\title{
Sonoluminescence: Coupling to an Applied Magnetic Field
}

\author{
B. A. DiDonna, T. A. Witten, and J. B. Young \\ Department of Physics and James Franck Institute, University of Chicago, \\ Chicago,Illinois 60637
}

\begin{abstract}
We investigate several means of coupling between a sonoluminescing bubble and an applied magnetic field. Recent experiments show a strong quadratic dependence between the forcing pressures required for stable sonoluminescence and magnetic field amplitude. However, all coupling mechanisms calculated here for comparable magnetic fields involve energies no more than one percent the mechanical energy of bubble collapse. We conclude that the applied field must influence the system though its effect on some parameter which the bubble motion depends upon very sensitively. A few such mechanisms are suggested.
\end{abstract}

PACS: 78.60.Mq, 47.65.+a, 67.55.Fa

Key words: Sonoluminescence, Magnetohydrodynamics, Hydrodynamics

\section{Introduction}

Recent experiments [1] show that magnetic fields affect sonoluminescence. Sonoluminescence is the emission of light during the collapse of a bubble in a liquid subjected to an oscillating pressure field. [2-6] Young et al. [1] found that the presence of a uniform magnetic field increased the pressure amplitude for both the onset of stable sonoluminescence and the ultimate destruction of the bubble at high driving forces. Both thresholds shifted approximately quadratically in magnetic field strength, scaling by 1 atm per $(20 \mathrm{~T})^{2}$. This compares with typical driving forces of around $1 \mathrm{~atm}$ in the absence of a magnetic field.

Evidently fields of the order of 10 Tesla are enough to cause substantial changes in the emitted light. This implies a significant coupling of the magnetic field energy with the other energies in the collapsing, light-emitting bubble-kinetic 
energy and compressional energy. The detailed calculations of zero-field bubble collapse by $\mathrm{Wu}$ and Roberts [7] provide the quantitative basis for our estimates. From these calculations and previous experiments [2-6] a commonlyaccepted scenario of the luminescence emerges. Under optimal conditions a bubble is trapped at the antinode of a sinusoidal acoustic field near the center of a driven resonant acoustic chamber. During a large part of the acoustic cycle, the bubble is unstable against expansion. It expands from a radius of roughly 1 micron to a radius of thirty microns or more. Ultimately the ambient pressure becomes sufficiently positive that this large bubble collapses: the surface accelerates inward under the unbalanced external pressure. This inward motion continues past the point of force equilibrium; the inertia of the external liquid continues to compress the bubble. The collapse continues until the interior gas reaches extreme conditions. It becomes hot enough to ionize. Later, the inward velocity exceeds the thermal velocities inside the bubble and an imploding shock wave is launched when bubble radius is a fraction of a micron. The emitted light is thought to come from the highly compressed center of this shock-wave region, over a timescale of picoseconds or less. Ultimately the inward motion is stopped by the large excess of outward force and then the bubble re-expands. This re-expansion occurs faster than the acoustic period; thus the ambient pressure during expansion is still strong enough to cause collapse. This results in a sequence of small collapse bounces after the initial one. Optimal sonoluminescence requires specific choices of gas dissolved in the water. Bubble trapping can be observed without collapse; likewise trapping and collapse can be observed without substantial light emission.

In this paper we survey the potential coupling mechanisms between the magnetic field energy and other forms of energy. We begin with the sonoluminescent plasma, the site of strongest interaction. After estimating the strength of the coupling here, we consider regions progressively more distant from the center. Some have more than one possible coupling mechanism. Our estimates do not claim to cover new theoretical ground. The interaction of magnetic fields with imploding matter is a well explored subject in astrophysical [8] and nuclear physics [9] contexts. Our aim here is to evaluate these well-known effects under the specific experimental conditions where sonoluminescence is observed. The main coupling of the external field to the ionized interior of the emitting bubble arises through its conductivity. Plausible estimates of the conductivity and conducting volume allow one to judge how much the plasma modifies the field. Beyond this conducting volume is a neutral region within the bubble but outside the shock front. We consider possible conductivitybased coupling in this region. Proceeding outward to the bubble surface, we consider the effects of surface charge and of diamagnetic contrast. Beyond the surface, in the bulk liquid, we consider the possibility of Seebeck currents.

All calculations given here are based on the simulated bubble collapse detailed in $\mathrm{Wu}$ and Roberts [7]. Although $\mathrm{Wu}$ and Roberts base their calculations on 
a bubble of nitrogen gas, we assume an argon gas bubble, since argon is very effective at luminescing. The choice of gas is for concreteness, and does not significantly affect numerical results.

The magnetic field interaction energies must be compared to the zero-field energy of the collapse. For all these potential effects the simplest order-ofmagnitude estimates are sufficient to judge their importance. The hydrodynamic bubble collapse powers itself, so that the bubble has absorbed all the mechanical energy it will receive from the acoustic field at the point when it reaches its maximum radius, just before it implodes. The energy of the collapse is then (assuming that the bubble expands adiabatically, i.e. $p V^{\gamma}$ is constant, for $\gamma=1.4) U=\int P d V$. For the bubble cycle simulated in [7], the maximum bubble radius is $30 \mu \mathrm{m}$, so the energy of collapse is 0.03 ergs, or $3 \mathrm{~nJ}$.

An independent method of calculating the collapse energy involves the surface speed of the bubble in the final moments of implosion, when all the collapse energy is in the kinetic energy of the inward rushing water. Assuming the water is incompressible and therefore the velocity of the fluid scales as $1 / r^{2}$, the kinetic energy in the water is $\int_{R}^{\infty} \frac{1}{2} \rho_{w} v^{2}(r) d V=2 \pi \rho_{w} R_{s}^{3} v_{s}^{2}$, where $R_{s}$ and $v_{s}$ are the surface radius and speed, respectively. At the point when the bubble reaches a radius of one micron, the surface speed of the bubble wall approaches $10^{5} \mathrm{~cm} / \mathrm{s}$, so the kinetic energy of the water is about 0.06 ergs. Our two methods of calculating the collapse energy agree to a factor of two, which gives us a good level of confidence that this is the energy scale typical of the system.

In all cases the mechanisms we investigated appear to be one percent or smaller perturbations to the mechanical energy of bubble collapse. We therefore conclude that the applied magnetic field must be changing some sensitive parameter in the hydrodynamic equations which we cannot analyze with our simple energy scale comparisons. A possible effect of this nature is discussed at the end of this paper.

\section{Interior Plasma}

\subsection{Flux Trapping}

We first considered the interaction of the external field with the hot plasma formed just before the light burst. The coupling of magnetic fields to shock waves in a collapsing bubble has been studied in greater detail elsewhere [10] for both the high and low limit of magnetic Reynolds number. We content ourselves here to make only energy estimates based on our "typical" sonolu- 
minescence parameters.

The collapsing plasma compresses the magnetic flux and the collapse is thereby inhibited. The final collapse and formation of a shock wave take place over a timescale of $10^{-10}$ seconds and a length scale of 0.3 microns. Temperatures can range from $10^{3}$ to $10^{7} \mathrm{~K}$. For flux trapping to be an important effect, two conditions must be met: the flux must remain trapped for a time comparable to the collapse time and the work required to compress the field lines must be comparable to the energy in the bubble without magnetic field.

First we calculate the magnetic diffusion time and compare it to the collapse time. Flux trapping requires that the diffusion time be longer than the collapse time. Jackson [11] gives the time constant of magnetic field diffusion as

$$
\tau_{D}=\frac{4 \pi \sigma L^{2}}{c^{2}}
$$

where $\sigma$ is the conductivity and $\mathrm{L}$ is the characteristic length scale. We model the conductivity of the plasma in Drude style, giving a DC conductivity $\sigma=$ $n e^{2} \tau / m_{e}$ where $\tau$ is now the mean-free scattering time for an electron in the material and $n$ is the density of conduction electrons.

As a first approximation which should overestimate the time constant and therefore the conductivity, we treat the free electrons as a Maxwell-Boltzman gas. The time constant is then given by the mean free path divided by the average velocity, $\tau=l /\langle v\rangle,\langle v\rangle \approx \sqrt{3 k_{B} T / m_{e}}$. The mean free path is $l \approx$ $1 /\left(\sigma_{o} N\right)$ where $\sigma_{o}$ is the atomic cross section (Coulombic) $\approx 16 \times 10^{-16} \mathrm{~cm}^{2}$.

Labeling $n=\mathcal{Z}(T, L) N$, with $\mathcal{Z}$ the average ionization of the atoms and $N$ the number density of atoms, we find

$$
\sigma=\frac{\mathcal{Z} e^{2}}{\sigma_{o} \sqrt{3 m_{e} k_{B} T}}
$$

Therefore the characteristic timescale is:

$$
\tau_{D}=\frac{4 \pi \mathcal{Z} e^{2}}{\sigma_{o} c^{2} \sqrt{3 m_{e} k_{B} T}} L^{2} \text { or } \tilde{\tau}_{D}=3 \times 10^{-3} \frac{\mathcal{Z}}{\sqrt{\tilde{T}}} \tilde{L}^{2}
$$

Here $\tilde{\tau}_{D}$ is the time constant in seconds, $\tilde{L}$ is the size of the region in $\mathrm{cm}$, and $\tilde{T}$ is the temperature in Kelvin.

If we take $\tilde{L}=3 \times 10^{-5} \mathrm{~cm}$, then $\tilde{\tau}_{D} \approx 3 \times 10^{-12} \mathcal{Z} / \sqrt{\tilde{T}}$. Considering a bubble of argon gas, even a massive electrical breakdown at $1000 \mathrm{~K}$ would only produce at most 8 electrons per atom and therefore a time constant of one picosecond. 
Better modeling of the ionization using Saha's equation [12] gives values of $\mathcal{Z} / \sqrt{\tilde{T}}$ on the order of 0.02 for temperatures between $10^{3}$ and $2 \times 10^{5} \mathrm{~K}$, which gives values of $\sigma$ on the order of $10^{16} \mathrm{~S}^{-1}$ and timescales on the order of $10^{-13}$ seconds, about 3 orders below the timescale of the sonoluminescing shock wave. The corresponding magnetic Reynolds number is $R_{M}=v \tau / L=0.25$, safely in the regime of diffusion dominated (untrapped) field motion.

Next we compare the magnetic field energy to the energy in the bubble. The field energy density for a $10 \mathrm{~T}$ magnetic field is $4 \times 10^{8} \mathrm{ergs} / \mathrm{cm}^{3}$, so if the plasma couples to the magnetic field when the bubble radius is 0.3 microns the bubble encloses a field energy of only $5 \times 10^{-5}$ ergs. Thus even if there is perfect coupling to the magnetic field, the additional field pressure is at most a small perturbation to the system.

\subsection{Lorentz Forces}

So it seems that the magnetic field is essentially unscreened by the ionized plasma. Accordingly, we consider the forces on the plasma caused by the unscreened field. Lorentz forces will result in a current transverse to the collapse velocity. In the frame of the collapsing plasma the particles experience an electric field $\boldsymbol{E}^{\prime}=\beta \times \boldsymbol{B}$ in Gaussian units, where $\beta \equiv v / c\left(=10^{-4}\right.$ in the plasma collapse). This results in a current density (transverse to the collapse velocity) of magnitude $\boldsymbol{J}=\sigma \boldsymbol{E}^{\prime}$. The current will drain energy from the system in two ways - it will dissipate energy through ohmic heating and store energy through its interaction with the external magnetic field. We calculate the energy of both interactions.

Ohmic heating will dissipate energy at a rate

$$
P=\int_{V} \boldsymbol{J} \cdot \boldsymbol{E} \approx \sigma \beta^{2} B^{2} V .
$$

1 It was suggested to the authors [13] that the change in the heat conductance of the plasma due to the addition of the magnetic field may be enough to inhibit the shock. To find the effect of the B field on the heat conduction by thermal electrons, we must compare the cyclotron radius of the electrons in the $10 \mathrm{~T}$ field to their mean free path inside the plasma. The Maxwell-Boltzman value for electron velocities at the plasma temperatures given above will approach $10^{8} \mathrm{~cm} / \mathrm{s}$. Classically, the cyclotron radius for an electron traveling at this velocity is $r=\frac{m_{e} v c}{e B} \approx 5 \times 10^{-5}$ $\mathrm{cm}$. With atomic number densities of up to $10^{22} \mathrm{~cm}^{-3}$ in the plasma core, the mean free path for electrons is only on the order of $10^{-8} \mathrm{~cm}$. So it seems that the heat conductivity contributed by electrons in the the plasma is not significantly affected by the application of the magnetic field. 
For a field of $10 \mathrm{~T}$ and a plasma bubble of radius 0.3 microns, the power dissipated is $P=10^{5} \mathrm{ergs} / \mathrm{sec}$ - if power were dissipated at this rate for the entire duration of the plasma $\left(10^{-10} \mathrm{~s}\right)$, the total energy lost in the plasma interaction would be less than $10^{-5}$ ergs. Thus Ohmic loss due to the plasmamagnetic field interaction has no significant effect on the bubble motion.

To find the interaction energy between the current and the applied magnetic field we calculate the magnetic moment of this current distribution, taking it to be mainly dipole by axial symmetry. Defining the $\mathrm{z}$ axis in the direction of the magnetic field, The net magnetic moment is

$$
\begin{aligned}
\boldsymbol{M} & \equiv \frac{1}{2 c} \int \boldsymbol{x}^{\prime} \times \boldsymbol{J} d^{3} x^{\prime} \\
& =\frac{\pi}{3} \frac{\sigma v B}{c^{2}} R_{p}^{4} \hat{z},
\end{aligned}
$$

where we have assumed that $v$ is essentially constant throughout the plasma. Here $R_{p}$ is the radius of the plasma bubble, 0.3 microns. The first order correction to the field energy $U=\frac{1}{2}(\boldsymbol{B}+\boldsymbol{M})^{2}$ due to the induced magnetic moment is then

$$
U=\boldsymbol{M} \cdot \boldsymbol{B}=\frac{\pi}{3}\left(\frac{\sigma v}{c^{2}}\right) R_{p}^{4} B^{2}
$$

For the plasma parameters given previously, the interaction energy amounts to $3 \times 10^{-7}$ ergs, which is much smaller than other energy scales in the sonoluminescence cycle. Thus it is clear that the hot plasma at the core of the sonoluminescing bubble does not couple to external magnetic field strongly enough to alter the local field or the gas dynamics significantly. We therefore move on to examine coupling in other regions of the fluid-bubble system.

\section{Non-Plasma Bubble Interior}

Another possible locus of the magnetic field effect is the region outside the plasma. Immediately outside the plasma is an un-ionized region of the bubble. Due to the scarcity of free charge in the region, we saw no likely prospect for a strong magnetic field effect there. The one possible source of current in this region is the large thermal gradient between the plasma core and the bulk fluid outside the bubble. However, this subject is treated later for currents outside the bubble, where it is shown that the thermal currents required for significant coupling are greater than any we would expect to find in a system this small. We therefore don't consider any magnetic field interactions in the non-plasma bubble interior. 


\section{Bubble Surface}

\subsection{Surface Currents}

There is a possibility of finding large currents confined to the surface of the bubble, since there will be a localized ion build-up at the gas-fluid interface. This greatly enhances the electrical conductivity in that region. As the surface of the collapsing bubble sweeps through the applied magnetic field, Lorentz forces will cause a current to flow around the bubble. We compute the energy associated with this current and compare it to the 0.03 ergs which the bubble absorbs from the sound field (c.f. Section (1)). In order to estimate the maximum possible coupling to the field, we estimate the maximum carrier density and conductivity of the surface.

To estimate the maximum effect of the magnetic field we treat the bubble surface as a room temperature plasma. A typical charge density for a fully ionized surface is $0.2 \mathrm{C} / \mathrm{m}^{2}$, or $1.25 \times 10^{14}$ (charge carriers) $/ \mathrm{cm}^{2}$ [14]. It would be generous to assume that the carrier density at our bubble surface corresponds to approximately twice the surface charge given above (ions and their counterions) contained in a thin shell surrounding the bubble surface. Because of the small volume and low temperature of the charged shell, we do not expect strong coupling to the magnetic field (flux trapping), so we confine these calculations to the limit of unscreened magnetic field. The mechanisms for energy loss are exactly the same as those calculated above for the interior plasma bubble - Ohmic loss and field interaction energy. In either case we must estimate the electrical conductivity at the surface.

Since the current is made up of ions (dissociated water or salts) migrating through the liquid, the ion mobility is controlled by viscous drag. The maximum mobility for a sphere in a viscous medium is $v / F=1 /(6 \pi \eta a)$, where in this case $\eta$ is the viscosity of water $\left(0.01 \mathrm{~g} \mathrm{~cm}^{-1} \mathrm{~s}^{-1}\right)$ and $a$ is the effective radius of the ions, which we take to be about $2 \AA$. Substituting this into the definition of conductivity, $\boldsymbol{j}=\sigma \boldsymbol{E}$, and assuming most carriers are singly ionized, so that $\boldsymbol{j}=n e \boldsymbol{v}$ and $\boldsymbol{F}_{\text {ion }}=e \boldsymbol{E}$, we find

$$
\sigma=\frac{n e^{2}}{6 \pi \eta a} .
$$

If we assume the current flowing over the surface of the bubble is confined to a shell of thickness $w \approx 10$ Aor less, then the local charge density $n$ is approximately $2.5 \times 10^{18}$ (charge carriers) $/ \mathrm{cm}^{3}$ and the conductivity is $10^{8} \mathrm{~s}^{-1}$ (for comparison, typical metallic conductivities are on the order of $10^{18} \mathrm{~s}^{-1}$ and the conductivity of pure water is $4.5 \times 10^{4} \mathrm{~s}^{-1}$ ). 
Using Eq.(4) to calculate the Ohmic dissipation, we find the power loss is $P=4 \pi \sigma(\dot{R} / c)^{2} B^{2} R^{2} w$. (Note that the $\sigma$ calculated above is proportional to $1 / w$ through its dependence on $n$, so the combination $\sigma w$ is independent of $w$ and the equations given here are valid for any $w \ll R$.) The maximum surface speeds are on the order of $10^{5} \mathrm{~cm} / \mathrm{s}$, the time averaged bubble radius over the period of the collapse will be less than 20 microns, and the collapse itself takes about $2 \mu \mathrm{s}$. So a bubble collapse in the presence of a $10 \mathrm{~T}$ external field would dissipate less than $10^{-11}$ ergs — nine orders of magnitude less than the mechanical energy in the bubble.

Next we calculate the energy in the dipole interaction between current and magnetic field. Starting from Eq.(5) we take the dipole moment of the thin conducting surface to be $M=\frac{4 \pi}{3} \frac{\sigma \dot{R} B}{c^{2}} R^{3} w \hat{z}$. The field interaction energy is then $U=\left(\frac{\sigma w \dot{R}}{c^{2}}\right) \frac{4 \pi}{3} R^{3} B^{2}$. The factor in parentheses is dimensionless and independent of $w$, the factor after it is proportional to the magnetic field energy enclosed by the bubble. In a $10 \mathrm{~T}$ field, the bubble encloses a field energy of about 1000 ergs at maximum radius (30 microns); however, the surface velocity at this point is about $10^{3} \mathrm{~cm} / \mathrm{s}$, so the term in parentheses is $10^{-17}$ and the total energy only $10^{-14}$ ergs. Later in the collapse surface speeds are on the order of of $10^{5} \mathrm{~cm} / \mathrm{s}$, but by this time the radius is 10 microns or less, so the total energy from above is at most $4 \times 10^{-17}$ ergs. This is nowhere near the 0.03 erg total energy of the collapsing bubble. From these numbers it is clear that the energy of the plamsa-field interaction at the bubble surface is no more than $10^{-9}$ of the nonmagnetic bubble energy.

\subsection{Diamagnetic Pressure}

A further source of magnetic effect is not through Lorentz forces but through the magnetic polarizability $\chi$ of the fluid. The diamagnetism of water must add an additional outward pressure to the bubble walls. The magnetic permeability is defined as $\mu=1+4 \pi \chi$. According to [15], the difference in $\chi$ across the surface adds a uniform outward pressure on the bubble wall of order $\Delta \chi B^{2}$. For pure water, $\chi=-7.2 \times 10^{-7}$ so the pressure on a bubble in water at 10 Tesla will be $\sim 3.6 \times 10^{3}$ dynes $/ \mathrm{cm}^{2}\left(3.6 \times 10^{-3} \mathrm{~atm}\right)$. The field will also create a non-uniform pressure at the bubble surface that goes as $(\Delta \chi)^{2}|\boldsymbol{B} \cdot \boldsymbol{n}|^{2}$, which in our case is seven orders smaller than the uniform component. (We neglect the susceptibility of the gas inside the bubble. When the bubble is at maximum radius, the gas inside is so dilute that the diamagnetic susceptibility of water would be greater by a factor of two than even the paramagnetic susceptibility of a pure $\mathrm{O}_{2}$ bubble. Since the results of Young et. al. were identical for pure argon bubbles, the susceptibility of the gas cannot be making any significant difference.) We note that this is a lower bound, since typical magnetic impurities in the water could increase its susceptibility and enhance 
this effect. The magnetic impurity concentration of the water used in Kang's experiment is not known.

The diamagnetic pressure given above should be compared with the pressure on the bubble wall at maximum radius, which, as noted in the introduction, is a good measure of the total energy deposited in the bubble by the acoustic field. In the simulation by $\mathrm{Wu}$ and Roberts, the pressure at maximum radius is $0.54 \mathrm{~atm}$, so the diamagnetic pressure perturbs the mechanical energy by one part in a hundred.

This small perturbation may be significant, since even a slight change in water pressure can cause a marked change in the concentration of dissolved gases in the bubble. It was shown in [16] that a $5 \%$ change in the ambient pressure of the surrounding water can decrease the SL light intensity by $200 \%$, due to the change in saturation concentration of argon gas in the fluid. The $\sim 1 \%$ pressure drop due to diamagnetism could well have similar large effects on the gas concentration. The gas solubility effect cannot be analyzed within the energy scale framework set out in this paper, so we leave the possibility as a subject of future investigation.

Fortunately, the susceptibility effect can be tested by adding a paramagnetic salt to the water. A 0.05 molar solution of manganese chlorate should balance the outward diamagnetic pressure with an inward paramagnetic pressure. A 5 molar solution should produce a magnetic field pressure 100 times stronger than that in pure water. The nature of the paramagnetic effect may be different, since the direction of the pressure drop is reversed. Also, the presence of dissolved salts will alter the gas solubilities even in the absence of field. This test remains to be done.

\section{Bulk Fluid}

\subsection{Thermally Induced Currents}

Though we expect no significant currents on the bubble surface, there may be more extended currents in the water outside the bubble. This region has only weak coupling to the field (relative to the plasma and the bubble surface). On the other hand, it has a large volume. How could the field couple to the matter in this region? If it is to couple via the Lorentz force, there must be electric currents. The most obvious source of such currents we anticipate is a thermal gradient. The occurrence of such currents is known as the Seebeck effect. Accordingly, we asked whether a thermally generated current in the surrounding water, interacting with the $B$ field via the Lorentz force, might 
convert a significant amount of the inward momentum of the collapsing bubble into angular momentum.

The Seebeck coefficient of our water would enable us to convert this temperature drop to an electric current. We do not know this coefficient for the water used in our studies. However, we can attack the problem from the other end. We ask what sort of outward electric current $I$ would be required to substantially alter the momentum of the water? If the current required for strong magnetic coupling is much greater than any current that could exist in the system, then the coupling between the actual Seebeck currents and the external magnetic field must be negligible. To estimate the order of magnitude of the current required to strongly affect the bubble collapse, we demand that the interaction of this current with the external magnetic field be enough to add a transverse momentum equal to all the inward momentum of the collapse. Remembering that a significant Seebeck current will only flow for a small period of time $\Delta t$ when there is a large temperature gradient, we set

$$
\int_{t_{o}}^{t_{o}+\Delta t} d t^{\prime} \int_{r>R} d^{3} r \boldsymbol{j}\left(r, t^{\prime}\right) \times \boldsymbol{B} \approx \rho_{\text {water }} \int_{r>R} d^{3} r v\left(r, t_{o}\right)
$$

Again we use liberal estimates so as to find the maximum possible effect. The greatest current should flow at the last moment of the hydrodynamic collapse, a period $\Delta t$ lasting about $1 \mathrm{~ns}$, when the temperature of the bubble core jumps by a few orders of magnitude. We consider for definiteness the current flowing in the region of fluid between the bubble surface and twice that radius. At this time in the collapse the typical bubble radius is around 1 micron, so we consider the current flowing radially outwards across a distance $\Delta r=1 \mu \mathrm{m}$, filling a volume $\Delta V \approx \frac{4 \pi}{3}\left(2 \times 10^{-6} \mathrm{~m}\right)^{3}$. Typical bubble surface speeds are on the order $v=10^{3} \mathrm{~m} / \mathrm{s}$. We can therefore approximate the equality in Eq.(8) by:

$$
\rho_{w} \Delta V v \approx I B \Delta r \Delta t
$$

With $\rho_{w}=10^{3} \mathrm{~kg} / \mathrm{m}^{3}$ and for a $10 \mathrm{~T}$ applied field, solving for the current gives $I=3 \times 10^{3}$ amps. Let us put a current of this magnitude in perspective by calculating the associated ohmic heating. Very pure water has a resistivity of $\rho \approx 2 \times 10^{5} \Omega$-m; relatively impure water solutions can have resistivities as low as $1 \Omega$-m. Using the lower resistivity, a current of this magnitude flowing across a distance of $\Delta r=1$ micron would encounter a total resistance of $R=\rho \Delta r /($ area of bubble $) \geq 2 \times 10^{4} \Omega$ and therefore dissipate $I^{2} R \Delta t \geq 2 \times 10^{9}$ ergs as heat. This is clearly more energy than is deposited in the bubble, and much more than we would expect to find localized in any area in the system. We conclude that magnetic perturbation via Seebeck currents must be negligible. 


\section{Conclusion}

Our survey of possible magnetic effects has not identified a mechanism with an associated energy comparable with that of the unperturbed bubble. It is clear, however, that the magnetic field couples directly to many of the parameters involved in the hydrodymanics of bubble motion. We can only conclude that although the field does not directly change any hydrodyamnic parameter by a factor of order unity, it must significantly change some parameter upon which the system depends very sensitively. Under this assumption, one possible explanation of the magnetic field's effect on the system is through its influence on the concentration of dissolved gasses inside the bubble. The influence of dissolved gas concentrations on SL has already been studied [16]; it would be relatively straightforward to perform a detailed calculation of the partial pressure dependence on applied magnetic field.

We would like to thank M. Brenner, S. Hilgenfeldt, W. Kang, and R.B. Laughlin for enlightening discussions and suggestions. This work was supported primarily by the MRSEC Program of the National Science Foundation under Award Number DMR-9400379

\section{References}

[1] J. B. Young, T. Schmiedel, W. Kang, Phys. Rev. Lett. 77 (1996) 4816.

[2] D. F. Gaitan, L.A Crum, J. Acoust. Soc. Am. (Suppl. 1) 87 (1990) S141.

[3] B. P. barber, S. J. Putterman, Nature (London) 352 (1991) 318.

[4] D. F. Gaitan, L. A. Crum, C. C. Church, R. Roy, J. Acoust. Soc. Am. 91 (1992) 3166 .

[5] R. Hiller, S. J. Putterman, B. P. Barber, Phys. Rev. Lett. 69 (1992) 1182.

[6] B. P. Barber, S. J. Putterman, Phys. Rev. Lett. 69 (1992) 3839.

[7] C.C. Wu, P.H. Roberts, Phys. Rev. Lett. 70 (1993) 3842.

[8] H. Alfven, Cosmical Electrodynamics, Clarendon Press, Oxford, 1950.

[9] H. Hora, Laser Plasma and Nuclear Energy, Plenum Press, New York, 1975.

[10] T. Chou, E.G. Blackman, Phys. Rev. Lett. 76 (1996) 1549.

[11] J.D. Jackson, Classical Electrodynamics, 2nd ed., Wiley, New York, 1975.

[12] V. E. Golant, A. P. Zhilinsky, I. E. Sakharov, Fundamentals of Plasma Physics, Wiley, New York, 1980. 
[13] R. B. Laughlin, private conversation.

[14] J.N. Israelachvili, Intermolecular and Surface Forces, Academic Press, New York, 1985.

[15] B. M. Berkovsky, V. F. Medvedev, M. S. Krakov, Magnetic Fluids, Engineering Applications Oxford University Press, New York, 1993.

[16] L. Kondic, C. Yuan, C. K. Chan, Phys. Rev. E. 57 (1998) R32. 